\title{
EL BRANDING INTERNO Y POSICIONAMIENTO EN UNA UNI- VERSIDAD PÚBLICA DE PUNO-PERÚ
}

\author{
INTERNAL BRANDING AND POSITIONING IN A PUBLIC UNIVERSITY OF PUNO-PERU
}

MSc. Roger Freddy Fernández Burgos

rfernandezb@unap.edu.pe, ORCID: 0000-0002-1309-5999

Mg. Alberto Magno Cutipa-Limache

acutipa@unap.edu.pe, ORCID: 0000-0001-8584-6424

MSc. Walker Ernesto Aragón Cruz

walkeraragon@unap.edu.pe, ORCID: 0000-0002-0139-2961

Lic. Rocio Norma Mamani Alanoca

chio.ma.1528@gmail.com, ORCID: 0000-0002-0476-1339

Lic. Eva Puma Quecaño

katy16000@hotmail.com, ORCID: 0000-0002-6756-9512

UNIVERSIDAD NACIONAL DEL ALTIPLANO, PUNO, PERÚ

Recibido: septiembre 08, 2020

Aceptado: octubre 28, 2020

DOI: https://doi.org/10.38147/invneg.v13i22.107

\section{Resumen}

El objetivo del estudio fue determinar la relación que existe entre el branding interno y el posicionamiento de la Escuela Profesional de Ciencias de la Comunicación Social de la Universidad Nacional del Altiplano (UNA) durante el año 2019. Es una investigación cuantitativa de diseño no experimental, transeccional. La muestra es no probabilística intencional a 35 colaboradores; se utilizó como instrumento un cuestionario. Los resultados revelan que existe una relación directa y estadísticamente positiva muy alta ( $r=87.2 \%$ y $R^{2}=76 \%$ ) entre la variable branding interno y el posicionamiento de la Escuela Profesional de Ciencias de la Comunicación Social de la UNA Puno. Es decir, que las características del branding interno, identidad de los colaboradores, el trabajo en equipo, la adecuada gestión de la comunicación de marca y la interiorización de la misión, visión y valores generan un posicionamiento favorable a la escuela.

Palabras clave: Branding interno, Identidad corporativa, Comunicación de Marca, Posicionamiento.

\begin{abstract}
The objective of the study was to determine the relationship between internal branding and the positioning of the Professional School of Social Communication Sciences of the National University of the Altiplano (UNA) during 2019. It is a quantitative investigation of non-experimental design, transectional. The sample is intentional non-probability to 35 collaborators; A questionnaire was used as an instrument. The results reveal that there is a very high direct and statistically positive relationship $(r=87,2 \%$ and $R 2=76 \%$ ) between the internal branding variable and the positioning of the UNA Puno Professional School of Social Communication Sciences. In other words, the characteristics of internal branding, the identity of the collaborators, teamwork, the proper management of brand communication and the internalization of the mission, vision and values generate a favorable position for the school.
\end{abstract}

Keywords: Brand Communication, Corporate identity, Internal Branding, Positioning. 


\section{Introducción}

En el mundo existen innumerables marcas, las empresas hacen todo lo necesario para lograr la diferenciación en relación con la competencia, por ello implementan acciones diversas para buscar su posicionamiento. Según Ríos (2014), el branding es el arte de concebir, edificar y posicionar una marca, interactuando con el público meta, con el propósito de conseguir un vínculo emocional. También, (Capriotti, 2009) es definida como la gestión, estructura, y comunicación de los beneficios de la compañía, es decir, es un proceso que necesita de un manejo adecuado que certifique la identidad corporativa reflejada en los valores y atributos que representan a la compañía. Además, la gestión de atributos de identidad de la organización y la comunicación al público objetivo tiene como propósito lograr la identificación, diferenciación y preferencia de la compañía.

Para Gómez (2016), el branding es una herramienta que permite gestionar de manera eficiente la identidad de una organización o marca, de forma tal que pueda estructurar las bases endógena, y hacer uso de las variables exógenas, para asegurar un buen posicionamiento en el mercado meta. El branding es una estrategia de posicionamiento que sí influye a la hora de comunicar mensajes de marca, porque ejemplo el caso de Ron Santa Teresa ha logrado avanzar posiciones importantes en el mercado de venezolano, es reconocida, preferida y con una identidad sólida, razón por la cual, a la hora de comunicar mensajes, su alcance es extenso. En la actualidad se fortalece como la empresa líder de su categoría debido a que es reconocida, percibida y preferida como una buena imagen de marca.

Regalado-Ortegón (2019), en su investigación indican que los gurús o autoridades intelectuales son profesionales que: confían en sí mismos, se actualizan constantemente, trabajan en equipo, tienen una alta capacidad de transmisión de conocimientos y buscan una permanente innovación. Los buenos profesores se hacen en el camino, se necesita de experiencia para llegar a ser un excelente docente, puesto que, de las buenas y malas experiencias enriquecen sus cualidades. Pero todo esto, no es suficiente, sino se crea una marca personal, en la cual se hagan evidentes las características mencionadas anteriormente y se puedan comunicar eficientemente a los públicos objetivo, para convertirse en profesionales y personas memorables. Es por ello, los profesionales de la docencia universitaria, que deseen constituir una marca personal robusta y de valor para sus públicos objetivos, deben tener en cuenta, las características identificadas en el presente documento, comunicando dicho valor a su entorno para que sean tenidos en cuenta, por los diferentes actores de su entorno y alcanzar así el éxito profesional.

Fernández (2015), encontró que promocionar éticamente todas las acciones que en materia de responsabilidad social interna y externa ejecute la empresa, por ejemplo, elaborar un mínimo de dos informes de responsabilidad al año y que se comunique a la sociedad los progresos de la empresa en los componentes de economía, social y ambiental. Estas labores fortalecerán una imagen efectiva por parte de la comunidad vinculada (stakeholders o grupos de intereses), acrecentarán la credibilidad de la empresa. En suma, mientras más acciones de responsabilidad social implicará reforzar e incrementar el posicionamiento de cualquier organización, así lo mismo a sus respectivas marcas en el mercado meta logrando la preferencia de los consumidores.

La teoría del gobierno corporativo se fundamenta en el planteamiento de que este promueve el fortalecimiento operacional de las organizaciones y a su vez mejora su reconocimiento. Ello se sostiene en el código de buen gobierno corporativo (Méndez-Beltrán \& Rivera-Rodríguez (2015), que implica, establecer un conjunto de medidas que garantice los estímulos pertinentes para una administración eficiente de las organizaciones (Santonja, \& Borges, 2005). Además, que genere un conjunto de beneficios para los inversionistas y también es importante la confianza que se da entre la gestión de una compañía y la comunidad vinculada, estableciendo el compromiso para los directivos de la difusión de información sobre la situación financiera real de la organización (Correa, Quintero, Gómez, \& Castro, 2020).

Montúfar-Ipanaqué (2018), en su investigación encontró que el problema principal de Transportes Franchess es el desinterés que ha mostrado la gerencia en trabajar la comunicación dentro de su empresa, es decir, una comunicación con la comunidad vinculada externos e internos. Al no tener un representante que planté las políticas de lineamiento de la identidad de la marca en la empresa, no existe una identidad con la que los colaboradores puedan sentirse identificados como parte de la organización. También, es necesario que la empresa cuente con un representante de la comunicación tanto externa como interna de la empresa. Es cierto que la organización ha operado supuestamente bien sin una estrategia establecida en un plan de comunicación durante más de 20 años. Sin embargo, si desea diferenciarse ahora de las demás empresas será determinante que haga algo diferente a lo que su competencia oferta. Para comenzar, lo correcto es empezar desde el interior de la organización con la comunidad vinculada más relevante y sus colaboradores, quienes son el pilar fundamental de la actividad que desarrolla.

Enrriquez (2018), determinó que en general, el desarrollo de branding de marca en los clientes de la empresa se desarrolla regularmente, debido a que un $71 \%$ de los clientes así lo manifiestan, mientras que un $27 \%$ de clientes consideran que se desarrolla plenamente y un $2 \%$ quienes consideran que no lo hace. Estos resultados generales permitieron determinar a la empresa a tener presencia no solamente ante sus clientes, sino también a personas que no son clientes frecuentes. En cuanto al desarrollo de la propuesta de branding, se desarrolló estrategia de creación de logo en base a la actividad 
económica a la que se dedica la empresa, la creación de un eslogan el cual refleja las ventajas competitivas de la empresa y proponer medios de promoción de los productos que ofrece la empresa.

En la actualidad las organizaciones públicas y particularmente las universidades han hecho muy poco por implementar estrategias para comunicar los valores y atributos del quehacer universitario, como no se conoce, no se tiene percepciones claras sobre la posición de la marca institucional en la mente de los usuarios y la comunidad vinculada. Especialmente en la Universidad Nacional del Altiplano Puno, en la Escuela Profesional de Ciencias de la Comunicación Social, el branding interno aun no es entendida en su real dimensión, sus directivos no consideran significativo gestionar la marca, la imagen, la identidad, el compromiso de sus colaboradores; como no se aplica estrategias de branding, no se tiene información para la toma de decisiones, tampoco se puede determinar efectivamente como esta herramienta puede contribuir en la mejora del posicionamiento.

Si bien es cierto la escuela gestiona una marca ya definida que la comunidad vinculada conoce, lo recuerdan, pero, no se tiene información en qué medida se encuentran, tampoco se conoce el grado de identificación de sus colaboradores, se observa que en la mayoría de ellos existe poca identificación, poco compromiso y poca motivación, ello redunda en el logro de objetivos institucionales.

Bajo el escenario planteado, el objetivo del estudio fue determinar la relación entre el branding interno y el posicionamiento de la Escuela Profesional de Ciencias de la Comunicación Social de la Universidad Nacional del Altiplano durante el año 2019.

\section{Material y método}

La investigación se desarrolla bajo el cuantitativa, al respecto Hernandez, (2014), sostienen que se usa la recolección de la data para validar o probar la hipótesis de investigación, apoyado en medidas numéricas para el análisis estadístico, formar patrones de comportamiento de fenómenos y comprobar teorías. El diseño de investigación fue no experimental y transeccional de tipo descriptivo - relacional, con el propósito de medir la relación de la variable de estudio branding interno, sus dimensiones identidad corporativa y comunicación de marca con la variable posicionamiento de la Escuela Profesional de Ciencias de la Comunicación UNAP, ubicado a las orillas del lago navegable más alto del mundo en un contexto en particular. La población de estudio es- tuvo conformada por el total de colaboradores, se aplicó el muestreo no probabilístico intencional a 35 docentes de la escuela profesional (Vara, 2012). La técnica aplicada fue la encuesta, se utilizó como instrumento un cuestionario de tipo escala Likert, donde cada pregunta tiene cinco alternativas de respuesta: Totalmente en desacuerdo $(T E D))=1$, En desacuerdo $(E D)=2$, Ni de acuerdo ni en descuerdo (NDANED) $=3$, De acuerdo (DA) $=4$, Totalmente de acuerdo $(T D A)=5$, ejecutándose el trabajo en el año 2018. El procesamiento de datos para la obtención de la información se desarrolló utilizando el software Microsoft Excel y el SPSS Statistics, cuya información se presenta en tablas y figura correspondientes.

\section{Resultados y discusión}

\subsection{Resultados}

Los resultados (tabla 1) evidencian que, en la Escuela Profesional de Ciencias de la Comunicación de la Universidad Nacional del Altiplano de Puno, el 42.9\% manifiestan que están de acuerdo en que la identidad corporativa es un elemento clave del branding, por lo que se debe dar la importancia del caso por parte de los directivos de la escuela. Asimismo, un importante número de colaboradores que representan el $25.7 \%$ manifiestan estar ni de acuerdo ni en descuerdo, es decir para ellos no es importante el branding. Adicionalmente en un número de 7 colaboradores que equivale al $20 \%$ indican estar en desacuerdo con el branding interno en la escuela profesional.

En relación con el posicionamiento de la escuela profesional se muestra que 19 colaboradores equivalente al $54 \%$ indican estar de acuerdo con el lugar que ocupa la escuela profesional en la mente de la comunidad vinculada; en cambio el $20 \%$ de los miembros colaboradores de la escuela manifiesta estar totalmente de acuerdo con la percepción de la imagen que tiene la escuela profesional.

Permitiendo concluir que en la Escuela Profesional de Ciencias de la Comunicación Social de la UNA Puno la mayoría de los miembros colaboradores creen que los atributos o características como la identidad, la contribución personal, la ejecución de sus actividades laborales, su empeño son elementos importantes del branding interno. Por su parte una gran mayoría de colaboradores 26 en total equivalente al 74\% (DA+TDA) indican que la escuela profesional tiene un buen posicionamiento, es decir, que los públicos interés tienen una buena percepción de la escuela profesional. 
Tabla 1.

Relación entre la dimensión de identidad corporativa y la variable posicionamiento

\begin{tabular}{|l|c|c|c|c|}
\hline \multirow{2}{*}{ Escala } & \multicolumn{2}{|c|}{ Identidad Corporativa } & \multicolumn{2}{c|}{ Posicionamiento de la Escuela } \\
\cline { 2 - 5 } & Frecuencia & $\%$ & Frecuencia & $\%$ \\
\hline TED & 2 & 5.7 & 1 & 3 \\
ED & 7 & 20.0 & 3 & 9 \\
NDANED & 9 & 25.7 & 5 & 14 \\
DA & 15 & 42.9 & 19 & 54 \\
TDA & 2 & 5.7 & 7 & 20 \\
\hline TOTAL & 35 & 100 & 35 & 100 \\
\hline
\end{tabular}

Nota. Totalmente en desacuerdo (TED), En desacuerdo (ED), Ni de acuerdo ni en descuerdo (NDANED), De acuerdo (DA) y Totalmente de acuerdo (TDA)

Fuente: Base de datos según encuesta aplicada.

Los resultados (tabla 2) evidencian que el $62.9 \%$ de los miembros colaboradores de la escuela profesional manifiestan que están de acuerdo con la gestión de la comunicación de marca, es decir, en un porcentaje mayor los colaboradores vienen desarrollando y experimentando un grupo importante de atributos y valores de forma apropiada, por ejemplo el nivel de compromiso de sus miembros es alto, por lo que se desprende el interés en asistir a las actividades como reuniones, seminarios en forma voluntaria.

Esta experiencia de atributos y valores personales, organizacionales deben examinase y perfeccionarse de tal modo que la comunicación de marca proyecte la imagen esperada de la escuela para que sea fácilmente recordada, valorada y reconocida. Por otro lado, el $25.7 \%$ de los colaboradores muestra una posición neutral al señalar que solo cumplen con sus funciones en comendadas, además que es un deber asistir a dichas actividades académicas.

En cambio, el $54 \%$ de los miembros colaboradores de la escuela profesional manifiestan que están de acuerdo con el posicionamiento actual de la escuela. Por lo tanto, podemos deducir que si bien es cierto están de acuerdo con dicha característica, ésta aún puede ser mejorada propiciando el desarrollo de nuevas estrategias de servicio, de calidad académica, entre otros. Por último, en la escuela un gran número de colaboradores valoran a los atributos de asistencia a reuniones, involucramiento y se informa de las acciones académicas como parte de sus responsabilidades laborales, las mismas que están siendo ejercidas de forma regular a buena.

\section{Tabla 2.}

Relación entre la dimensión comunicación de marca y la variable posicionamiento

\begin{tabular}{|l|c|c|c|c|}
\hline \multirow{2}{*}{ Escala } & \multicolumn{2}{|c|}{ Comunicación de marca } & \multicolumn{2}{c|}{ Posicionamiento de la Escuela } \\
\cline { 2 - 5 } & Frecuencia & $\%$ & Frecuencia & $\%$ \\
\hline TED & 1 & 2.9 & 1 & 3 \\
\hline ED & 2 & 5.7 & 3 & 9 \\
\hline NDANED & 9 & 25.7 & 5 & 14 \\
\hline DA & 22 & 62.9 & 19 & 54 \\
\hline TDA & 1 & 2.9 & 7 & 20 \\
\hline TOTAL & 35 & 100 & 35 & 100 \\
\hline
\end{tabular}

Nota. Totalmente en desacuerdo (TED), En desacuerdo (ED), Ni de acuerdo ni en descuerdo (NDANED), De acuerdo

(DA) y Totalmente de acuerdo (TDA)

Fuente: Base de datos según encuesta aplicada.

En los resultados (tabla 3 ) se muestran que el $54.3 \%$ de los miembros colaboradores manifiestan que están de acuerdo con la gestión del branding interno, es decir, los directivos de la escuela profesional se esfuerzan en instituir y promocionar la identidad corporativa. Para ello, son esenciales la ejecución de estrategias y táctica como vencer el escepticismo, la inseguridad, comprender el trabajo en equipo, dejando de lado las oposiciones contrarias y prevaleciendo el consenso. Además, las exigencias de la nueva ley universitaria demandan el cumplimiento de condiciones elementales básicas de calidad, por lo que, los miembros colaboradores de la escuela han interiorizado en la práctica la visión, la misión, valores corporativos y ante todo un potente y nueva personalidad de marca. 
En cambio, el $25.7 \%$ de los miembros colaboradores de la escuela profesional sostiene estar ni de acuerdo ni en descuerdo, es decir se mantienen en una posición neutral, el cual implica solamente el cumplimiento de sus funciones y compromisos según lo establecido en los instrumentos de gestión. Finalmente, en un número de 4 miembros colaboradores que representan el $11.4 \%$ del total encuestados manifiestan estar en desacuerdo con la gestión de branding interno.

\section{Tabla 3.}

Relación entre las variables de Branding Interno y el posicionamiento de la Escuela Profesional del Ciencias de la Comunicación Social UNAP.

\begin{tabular}{|l|c|c|c|c|}
\hline \multirow{2}{*}{ Escala } & \multicolumn{2}{|c|}{ Branding Interno } & $\begin{array}{c}\text { Posiciona- } \\
\text { miento de la } \\
\text { Escuela }\end{array}$ & \\
\cline { 2 - 5 } & Frecuencia & $\%$ & Frecuencia & $\%$ \\
\hline TED & 2 & 5.7 & 1 & 3 \\
\hline ED & 4 & 11.4 & 3 & 9 \\
\hline NDANED & 9 & 25.7 & 5 & 14 \\
\hline DA & 19 & 54.3 & 19 & 54 \\
\hline TDA & 1 & 2.9 & 7 & 20 \\
\hline TOTAL & 35 & 100 & 35 & 100 \\
\hline
\end{tabular}

Nota. Totalmente en desacuerdo (TED), En desacuerdo (ED), Ni de acuerdo ni en descuerdo (NDANED), De acuerdo (DA) y Totalmente de acuerdo (TDA)

Fuente: Base de datos según encuesta aplicada.

El coeficiente de correlación de Pearson, indica que existe una correlación positiva muy alta entre las variables branding interno y el posicionamiento de la Escuela y que estadísticamente es muy significativa con un $r=0.872$.

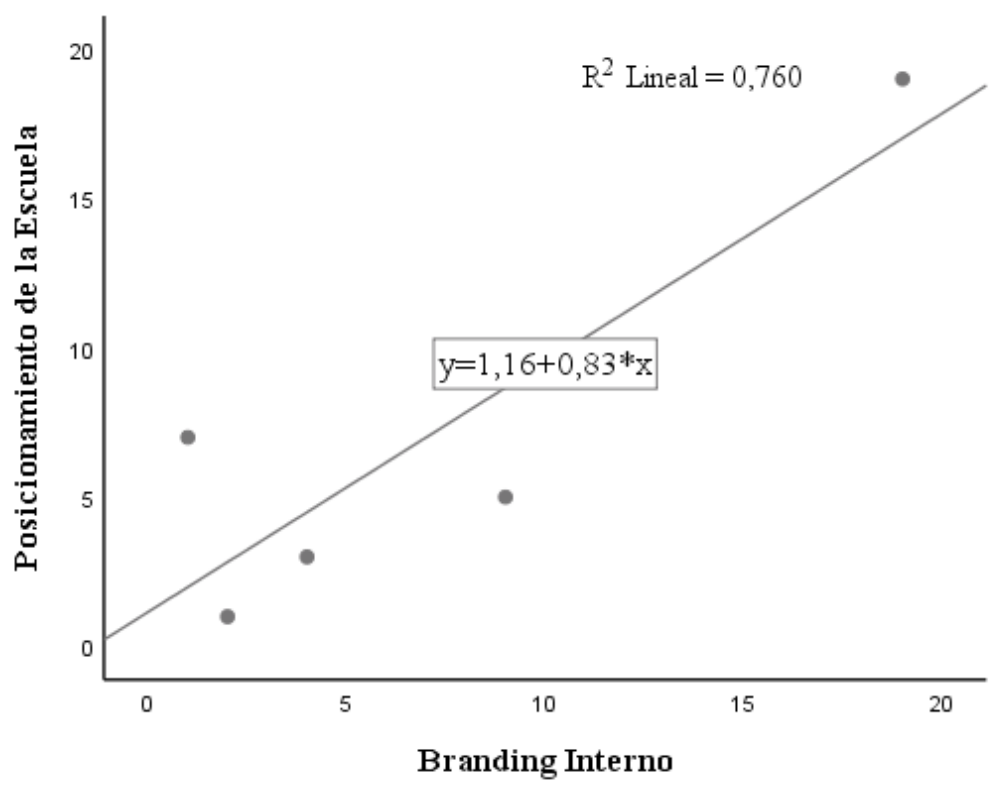

Figura 1. Dispersión del branding interno y el posicionamiento de la Escuela Profesional

En los resultados (figura 1) de la gráfica de dispersión, se observa que la relación entre ambas variables es directamente proporcional, es decir, a mejor branding interno le corresponde mejor posicionamiento, y a menor branding interno le corresponde menor posicionamiento. La ecuación $Y=1,16+0.83 X$ (donde: $Y=$ Posicionamiento de la Escuela, $X=$ Branding Interno) nos muestra la relación entre dichas variables, además el valor de R2 lineal que es el coeficiente de determinación, cuyo valor es de 0.76 , nos indica que dicha ecuación obtenida por regresión lineal representa el 76 $\%$ de los casos donde esta relación se cumple. 


\section{Investigación \& Negocios 132}

\subsection{Discusión}

En la presente investigación denominada el branding interno y su relación con el posicionamiento: un caso aplicado a una universidad pública se ha demostrado que, si existe una relación significativa entre ambas variables, con un nivel de correlación Pearson de $r=0,872$ lo cual es una relación positiva fuerte, por ende, se acepta la hipótesis alterna y se rechaza la nula. Esta información se puede corroborar con los antecedentes que se hacen mención, donde diversos autores en diferentes trabajos de investigación concluyen que existe relación entre el branding y el posicionamiento, y comparando con los resultados de la presente investigación, se puede afirmar que cuando se manejan estrategias adecuadas de identidad corporativa, comunicación de marca se logra un buen posicionamiento, deduciéndose que existe una relación entre estas dos variables estudiadas.

Se concluye según el análisis y resultados encontrados que el branding es una herramienta que gestiona de manera eficiente la identidad de una empresa y/o marca de forma tal que consiga estructurar las bases endógenas, y hacer uso de las variables exógenas, para garantizar un buen posicionamiento en el público objetivo. Gómez (2016), lo señalado nos permite destacar según el autor que es necesario tener conocimiento del uso y manejo de herramientas ya que están posibilitan estructurar y consolidar las estrategias necesarias para garantizar el tan ansiado posicionamiento. Méndez-Beltrán \& Rivera-Rodríguez (2015), manifiesta que aquellas organizaciones que gestionan de manera correcta sus stakeholders y fortalecen sus políticas de gobierno corporativo, han conseguido un mejoramiento en su posicionamiento; aquí el autor destaca que en la gestión pública y privada es necesario mantener una relación adecuada con las organizaciones de tu entorno. Al respecto, encontramos que, en la Universidad Nacional del Altiplano, escuela profesional de Ciencias de la Comunicación Social existe una relación directa y significativa entre las variables estudiadas, quiere decir que el branding interno tiene influencia en el posicionamiento de la Escuela.

Asimismo, Regalado-Ortegón (2019), señalan que los docentes universitarios como profesionales deben constituir una marca personal, que sea robusta y de valor para sus públicos objetivos, ellos deben comunicar dicho valor a los diferentes actores de su entorno. Los resultados hallados en la escuela profesional respecto al indicador identidad corporativa indican que dicho aspecto se viene ejecutando de manera regular, empero, que deben fortalecerse y repensarse algunas características de la identidad corporativa como algunos valores, creencias y filosofía en el trabajo que deben ser mejorados.

También Alcántar \& Arcos (2004), señalan que las universidades públicas deben adaptarse a los cambios que el mercado exige y que estas deben fomentar los mecanismos de cooperación, coordinación, consulta, promoción, imagen institucional y participación social; lo que causa impacto en el posicionamiento de la institución; lo aseverado concuerda con lo señalado en la ley Universitaria $\mathrm{N}^{\circ} 30220$, y las políticas de aseguramiento de la calidad educativa en la que se señala el cumplimiento de estándares de la calidad, características con las que cuenta la escuela profesional.

De manera similar Fernández, (2015), menciona que las empresas deben promocionar éticamente las acciones que en materia de responsabilidad social interna y externa realizan, lo que propiciara una imagen positiva hacia la comunidad vinculada, acrecentaran la credibilidad de la empresa, y como consecuencia de ello se reforzará e incrementará el nivel de posicionamiento de la organización, como de sus marcas ganando preferencias de sus consumidores. Partiendo de la premisa y comparando los resultados en la Escuela Profesional de Ciencias de la Comunicación existen características como la identidad, el aporte personal, el ejercicio de la labor elementos que están siendo conducidos de manera aceptable, ya que la escuela cuenta con la certificación de ser una escuela acreditada por SUNEDU, lo que le permite un buen posicionamiento en el ámbito externo.

En esta misma línea Fornelli \& Sánchez (2013), indican que el branding es como el cimiento en la estructura de las empresas y que en el proceso de construcción de la marca se tiene que fortificar las áreas externas e internas de la empresa lo que permite que un consumidor tenga una imagen favorable de ellos; esto confirma los resultados encontrados para la escuela profesional ya que indican estar de acuerdo con la gestión de comunicación de marca, lo que evidencia la mejora de un conjunto de prácticas de atributos y valores, es decir se ha reformado el nivel de compromiso, asistencia a las actividades como reuniones, seminarios de manera voluntaria. Estos atributos y valores personales, organizacionales han fortalecido y generado que la marca escuela profesional de Ciencias de la Comunicación sea fácilmente recordada, valorada y reconocida por la comunidad vinculada.

Castillo, Carrillo-Durán \& Luengo-Chávez (2019), para el caso de una cadena hotelera indican que para llegar a los stakeholders y generar experiencias que conlleven tanto a la diferenciación de la cadena como el logro de ventajas competitivas sostenibles en el mercado, es preciso que se comunique mensajes de marca que se respalden en los recursos intangibles primordiales con los que dispone (identidad corporativa, responsabilidad social corporativa y reputación corporativa). Por tanto, la marca debería fundamentarse el contenido apoyado en aquellos elementos intangibles que la sostienen y la diferencian, a través de la comunicación en integral y de la comunicación online en particular. Lo señalado, contraviene a lo encontrado en la investigación, que por ser una organización de carácter público evidentemente no desarrolla ni comunica estrategias, acciones concretas de los recursos intangibles. 


\section{Investigación $\&$ Negocios 133}

Se sugiere que los directivos de la universidad, directivos de la escuela, docentes y alumnos muestren mayor interés por la incorporación de estrategias novedosas de mercado que posibiliten mejorar el posicionamiento de organizaciones de carácter público como lo señalan Arteaga, Pianda \& Sandoval (2019).

\section{Conclusiones}

Existe una relación positiva muy alta $(r=0.872)$ entre el branding interno y el posicionamiento de la Escuela Profesional de Ciencias de la Comunicación Social de la Universidad Nacional del Altiplano Puno con un coeficiente de determinación de Pearson de $\mathrm{R}^{2}=0.76$ lo que equivale al $76 \%$. Los resultados nos indican que $54.3 \%$ de los colaboradores están de acuerdo con el branding interno, es decir, que los directivos están haciendo esfuerzos en mantener y desarrollar la identidad en sus colaboradores. Asimismo, se evidencia mayor cohesión entre los colaboradores, trabajo en equipo y se ha interiorizado la visión, la práctica de valores y sobre todo una nueva personalidad en la marca, esto ha generado mayor posicionamiento.

Por otro lado, existe una relación positiva y alta $(r=0.778)$ entre la dimensión de la identidad corporativa y el posicionamiento en la Escuela Profesional de Ciencias de la Comunicación Social de la Universidad Nacional del Altiplano Puno con un coeficiente de determinación de Pearson de $\mathrm{R}^{2}=0.61$ lo que equivale al $61 \%$. Los resultados evidencian que el $42.9 \%$ de los colaboradores están de acuerdo con la identidad corporativa, por lo que debe ser considerado como un elemento importante por parte de los directivos en los documentos de gestión estratégica para desarrollar una venta diferencial.

También, existe una relación positiva y muy alta $(r=0.901)$ entre la dimensión de la comunicación de marca y el posicionamiento en la Escuela Profesional de Ciencias de la Comunicación Social de la Universidad Nacional del Altiplano Puno con un coeficiente de determinación de Pearson de $\mathrm{R}^{2}=0.82$ lo que equivale al $82 \%$. Los resultados nos indican que el $62.9 \%$ de los colaboradores están de acuerdo con la gestión de comunicación de marca lo que evidencia la mejora de un conjunto de prácticas de atributos y valores, se ha mejorado el nivel de compromiso, asistencia a actividades como reuniones, seminarios es voluntaria. Estos atributos y valores personales e institucionales han fortalecido y promovido que la marca Escuela Profesional de Ciencias de la Comunicación Social sea fácilmente recordada, valorada y reconocida por la comunidad vinculada.

\section{Agradecimientos}

Este trabajo es producto de una investigación de pregrado: Análisis del Branding Interno y el posicionamiento de los docentes de la Escuela Profesional de Ciencias de la Comunicación Social de la UNA Puno.

\section{Referencias}

Alcántar, V. M., \& Arcos, J. L. (2004). La vinculación como instrumento de imagen y posicionamiento de las instituciones de educación superior. Revista Electrónica de Investigación Educativa, 6(1), 01-12. Recuperado de https:// redie.uabc.mx/redie/article/view/92

Arteaga, A. L., Pianda, E. M., \& Sandoval, X. A. (2019). City Branding como estrategia de mercado para promocionar el turismo en la ciudad de Ipiales. Tendencias, 20(1), 22-52. https://doi.org/10.22267/rtend.192001.106

Capriotti, P. (2009). Branding Corporativo. Fundamentos para la gestion estrategica de Identidad Corporativa. Santiago, Chile: Andros Impresores.

Castillo, A., Carrillo-Durán, M. V., \& Luengo-Chávez, G. (2019). Brand communication of hotel chains through their websites: Proposed model for their management. Palabra Clave, 22(2), 1-33. https://doi.org/10.5294/pacla.2019.22.2.11

Correa Mejía, D. A., Quintero Castaño, J. D., Gómez Orozco, S., \& Castro Castro, C. M. (2020). El gobierno corporativo, un pilar indispensable para el desempeño financiero. (Spanish). Universidad \& Empresa, 22(38), 40. http://dx.doi. org/10.12804/revistas.urosario.edu.co/empresa/a.6975

Enrriquez, E. R. (2018). Estrategias de branding en una empresa de representación y comercialización de insumos serigráficos de Chiclayo 2018. (Tesis de pregrado, Universidad Señor de Sipan). Recuperado de http://200.60.28.13/ bitstream/handle/uss/5540/Enrriquez\%20Tapia\%20Evelyn\%20Rocio.pdf?sequence=1\&isAllowed=y

Fernández, A. (2015). Responsabilidad social para el pocisionamiento de las marcas en el sector textil. Revista del Centro de Investigación de Ciencias Administrativas y Gerenciales, 12(2), 146-159. Retrieved from http://ojs.urbe.edu/ index.php/cicag/article/view/1777/1696

Fornelli, A., \& Sánchez, J. O. (2013). Branding para la pequeña y mediana empresa. Cultura Científica y Tecnológica, 2(49), 50-53. Recuperado de http://erevistas.uacj.mx/ojs/index.php/culcyt/article/viewFile/183/177 


\section{Investigación Negocios $134^{134}$}

Gómez, E. (2016). Branding como estrategia de posicionamiento y su influencia en la comunicación de marca Caso : Ron Santa Teresa. (Tesis de pregrado, Universidad Central de Venezuel). Recuperado de https://s3.amazonaws.com/academia.edu.documents/56230312/Tesis_UCV_2.pdf?response-content-disposition=inline $\% 3 B \% 20$ filename\%3DTesis_UCV.pdf\&X-Amz-Algorithm=AWS4-HMAC-SHA256\&X-Amz-Credential=AKIAIWOWYYGZ2Y53UL3A\%2F20200317\%2Fus-east-1\%2Fs3\%2Faws4_request\&X-Amz-Date=20200317T035922Z\&X-Amz -Expires $=3600 \& X-A m z-S i g n e d H e a d e r s=h o s t \& X-A m z-S i g n a t u r e=1122 b b 2 b 0 e c 534533422415201521 \mathrm{~d} 4828 a b c 05 d-$ c0071646f57d76cab9ab952e

Hernandez, F. C. (2014). Metodologia de Investigacion. Mexico D.F.: Mc Graw Hill.

Méndez-Beltrán, J. A., \& Rivera-Rodríguez, H. A. (2015). Relación entre gobierno corporativo y posicionamiento organizacional: instituciones de educación superior en América Latina. Educación y Educadores, 18(3), 435-455. https:// doi.org/10.5294/edu.2015.18.3.4

Montúfar-Ipanaqué, E. (2018). Propuesta para el desarrollo de una identidad corporativa y estrategia de branding interno: caso Transportes Franchess S.C.R.L. (Tesis de pregrado, Universidad de Piura). Recuperado de https://pirhua. udep.edu.pe/bitstream/handle/11042/3358/TSP_INF_017.pdf?sequence=1\&isAllowed=y

Regalado-Ortegón, A. (2019). Branding Personal y Creación de Gurús Académicos. Cuadernos Latinoamericanos de Administración, 14(26). https://doi.org/10.18270/cuaderlam.v14i26.2289

Vara, A. (2012). Siete pasos para hacer una tesis exitosa. Un método efectivo para las Ciencias Empresariales. Lima, Perú: Instituto de Investigación Facultad de Ciencias Administrativas y Recursos Humanos.

Ríos, N. G. (2014). Percepción de los estudiantes de educación superior sobre el posicionamiento de marca mediante la gestión del branding, aplicado en el concurso de Desafío SEBRAE, durante el 2012. Revista de Investigación Universitaria, 3(2), 11-22. https://doi.org/https://doi.org/10.17162/riu.v3i2.516

Santonja, A. O., \& Borges, A. M. (2005). Teoría y práctica del buen gobierno corporativo. Marcial Pons. Recuperado de http://www.villafane.com/wp-content/uploads/2015/11/Cap-8_2005_Teoria-y-practica-del-buen-gobierno-corporativo_Aldo-Olcese.pdf 